This item was submitted to Loughborough's Research Repository by the author.

Items in Figshare are protected by copyright, with all rights reserved, unless otherwise indicated.

\title{
Solitary waves propagating over variable topography
}

PLEASE CITE THE PUBLISHED VERSION

LICENCE

CC BY-NC-ND 4.0

\section{REPOSITORY RECORD}

Grimshaw, Roger H.J.. 2019. "Solitary Waves Propagating over Variable Topography". figshare. https://hdl.handle.net/2134/2330. 


\section{Solitary waves propagating over variable topography}

Roger Grimshaw

Loughborough University, Loughborough, LE11 3TU, UK

R.H.J.Grimshaw@lboro.ac.uk

\section{Introduction}

Solitary water waves are long nonlinear waves that can propagate steadily over long distances. They were first observed by Russell in 1837 in a now famous report [26] on his observations of a solitary wave propagating along a Scottish canal, and on his subsequent experiments. Some forty years later theoretical work by Boussinesq [8] and Rayleigh [25] established an analytical model. Then in 1895 Korteweg and de Vries [21] derived the well-known equation which now bears their names. Significant further developments had to wait until the second half of the twentieth century, when there were two parallel developments. On the one hand it became realised that the Korteweg-de Vries equation was a valid model for solitary waves in a wide variety of physical contexts. On the other hand came the discovery of the soliton by Kruskal and Zabusky [27], with the subsequent rapid development of the modern theory of solitons and integrable systems.

In this chapter, we are mainly concerned with the behaviour of solitary waves as they propagate through a variable medium, with a particular emphasis on water waves over variable topography. But, first, we consider the well-known situation when the background medium is uniform. Solitary waves owe their existence to a balance between nonlinearity and wave dispersion. When both these effects are weak, the leading order model evolution equation is the Korteweg-de Vries (KdV) equation,

$$
A_{t}+c A_{x}+\mu A A_{x}+\delta A_{x x x}=0 .
$$

Here $A(x, t)$ is the amplitude of the relevant wave mode, which is assumed to be propagating in the $x$-direction, $c$ is the speed of a linear long wave, $\mu, \delta$ are the coefficients of the quadratic nonlinear term, and the third-order linear dispersive term, respectively, and, like $c$ depend on the particular physical system being considered. The leading terms here are the first two, which describe a linear long wave propagating with speed $c$; relative to these dominant 
terms, the remaining two terms describe a balance between weak nonlinear steepening and weak linear dispersion. It is precisely this balance which describes solitary waves. For water waves propagating over a constant depth $h$ it is well known that [21]

$$
c=\sqrt{g h}, \quad \mu=\frac{3 c}{2 h}, \quad \delta=\frac{c}{6 h^{2}} .
$$

where $A$ is the surface elevation above the undisturhed depth $h$. The KdV equation (1) is integrable (see, for instance, [1], [2], [4]) and many fascinating properties follow.

However, our main concern here is with the one-phase periodic travelling wave solutions of the $\mathrm{KdV}$ equation (1), the so-called cnoidal waves

$$
\begin{aligned}
A & =a\left\{b(m)+\mathrm{cn}^{2}(\gamma \theta ; m)\right\}+d, \\
\text { where } \quad \theta & =k(x-V t), \\
b(m) & =\frac{1-m}{m}-\frac{E(m)}{m K(m)}, \quad \mu a=12 m \delta \gamma^{2} k^{2}, \\
\text { and } \quad V & =c+\mu d+\frac{\mu a}{3}\left\{\frac{2-m}{m}-\frac{3 E(m)}{m K(m)}\right\} .
\end{aligned}
$$

Here $c n(x ; m)$ is the Jacobian elliptic function of modulus $m, 0<m<1$ and $K(m), E(m)$ are the elliptic integrals of the first and second kind. The amplitude is $a$, the mean value of $A$ over one period is $d$, while the spatial period is $2 K(m) / \gamma k$. But since we can choose the phase $\theta$ so that $A$ is $2 \pi$ periodic in $\theta$ we see that $\gamma=K(m) / \pi$. This periodic travelling wave (3) contains three free parameters; we take these to be the amplitude $a$, the mean level $d$ and the modulus $m$, so that equations $(5,6)$ then determine $k, V$ respectively.

As the modulus $m \rightarrow 1$, this becomes a solitary wave, since then $b \rightarrow 0$ and $\operatorname{cn}^{2}(x) \rightarrow \operatorname{sech}^{2}(x)$; in this limit $\gamma \rightarrow \infty, k \rightarrow 0$ with $\gamma k=K \Gamma$ held fixed. The outcome is the well-known solitary wave solution

$$
\begin{aligned}
A & =a \operatorname{sech}^{2}(\Gamma(x-V t)), \\
\text { where } \quad V & =c+\frac{\mu a}{3}=4 \delta \Gamma^{2} .
\end{aligned}
$$

On the other hand, as $m \rightarrow 0, \gamma \rightarrow 1 / 2$, and (3) reduces to sinusoidal waves of small amplitude $a \sim m$ and wavenumber $k$.

Many studies of weakly nonlinear long waves have used the KdV equation (1) or similar model equations with constant coefficents. However, particularly in the case of water waves in the coastal oceans, or for internal solitary waves propagating over the continental slope and shelf (see, for instance, [5]), [6], [16], [7]), there is a need to take account of the variation of the background medium in the wave propagation direction. In that case, the constant-coefficient equation (1) may be replaced by a variable-coefficient Korteweg-de Vries (vKdV) equation 


$$
A_{t}+c A_{x}+\frac{c Q_{x}}{2 Q} A+\mu A A_{x}+\delta A_{x x x}=0,
$$

Here $A(x, t)$ is again the amplitude of the wave, but now the speed $c$ and the coefficients $\mu, \delta$ depend on $x$. The coefficient $c$ is the relevant linear long wave speed, and $Q$ is the linear magnification factor, defined so that $Q A^{2}$ is the wave action flux. The vKdV equation was derived by Johnson [19] for water waves, where $Q=c$, and by Grimshaw [13] for internal waves (for recent reviews, see [5], [6]). The derivation assumes the usual KdV balance that the amplitude $A$ has the same order as the dispersion, measured by $\partial^{2} / \partial x^{2}$, and in addition assumes that the waveguide properties (i.e. the coefficients $c, Q, \mu, \lambda$ ) vary slowly so that $Q_{x} / Q$ for instance is of the same order as the dispersion. In section 2 we shall give a brief description of the derivation of (9) for water waves.

As the first two terms in (9) are again the dominant terms, it is useful to make the transformation

$$
\eta=\sqrt{Q} A, \quad \tau=\int^{x} \frac{d x}{c}, \quad \xi=\tau-t .
$$

Substitution into (9) yields, to the same order of approximation as in the derivation of (9),

$$
\begin{gathered}
\eta_{\tau}+\alpha \eta \eta_{\xi}+\beta \eta_{\xi \xi \xi}=0 \\
\alpha=\frac{\mu}{c \sqrt{Q}}, \quad \beta=\frac{\delta}{c^{3}} .
\end{gathered}
$$

Here the coefficients $\alpha, \beta$ are functions of $\tau$ alone. Note that although $\tau$ is a variable along the spatial path of the wave, we shall subsequently refer to it as the "time". Similarly, although $\xi$ is a temporal variable, in a reference frame moving with speed $c$, we shall subsequently refer to it as a "space" variable. The following sections are concerned with the derivation and with the solutions of equation (11)

\section{Derivation of a variable coefficient Korteweg-de Vries equation}

For simplicity, we shall describe the derivation of the variable coefficient KdV equation (9) for the main case of interest here, namely water waves propagating over variable topography. We consider a one-dimensional wave field so that the free-surface is represented by $z=\zeta(x, t)$ for an incompressible, inviscid fluid with constant density $\rho$, occupying the region $-h(x)<z<\zeta$ where the undisturbed depth $h(x)$ varies with $x$. The velocity field is $\mathbf{u}=(u, w)$ and can be assumed to be irrotational, so that $\mathbf{u}=\nabla \phi$, where $\phi(x, z, t)$ satisfies Laplace's equation

$$
\phi_{x x}+\phi_{z z}=0 .
$$


At the rigid bottom,

$$
w+u h_{x}=0 \quad \text { at } \quad z=-h .
$$

At the free surface, the flow must satisfy two conditions

$$
\begin{aligned}
\zeta_{t}+u \zeta_{x} & =w \quad \text { at } \quad z=\zeta, \\
\phi_{t}+\frac{|\mathbf{u}|^{2}}{2}+g \zeta=0 & \text { at } \quad z=\zeta .
\end{aligned}
$$

The first of these is the kinematic condition, and the second is the condition for constant pressure, where the Bernoulli relation has been used.

In order to obtain the vKdV equation (9) we shall use a multi-scale asymptotic expansion. This is a versatile approach and can be adapted to many other situations. Thus we introduce a small parameter, $\epsilon<<1$ measuring linear wave dispersion, and assume the usual KdV balance where the amplitude scales with $\epsilon^{2}$. We then rescale the horizontal coordinate and the time, so that

$$
X=\epsilon x, \quad T=\epsilon t,
$$

and seek an asymptotic expansion of the form

$$
\zeta=\epsilon^{2} \zeta^{(1)}(X, T)+\epsilon^{4} \zeta^{(2)}(X, T)+\cdots .
$$

There is a similar expansion for the other fluid variables. At the same time, we assume that the depth varies slowly on a spatial scale of $\epsilon^{-3}$, so that we may formally write $h=h(\chi)$ where $\chi=\epsilon^{2} X$.

It is convenient to define the depth-averaged mean flow

$$
U(X, T)=\frac{1}{h+\zeta} \int_{-h}^{\zeta} u(X, T, z) d z .
$$

Then it is readily shown that conservation of mass implies that

$$
\zeta_{T}+(U(h+\zeta))_{X}=0 .
$$

At the leading order we get

$$
\begin{aligned}
& \zeta_{T}^{(1)}+h U_{X}^{(1)}=0, \\
& U_{T}^{(1)}+g \zeta_{X}^{(1)}=0 .
\end{aligned}
$$

The general solution of this system is the sum of a wave propagating in the positive $X$-direction with speed $c$, and a wave propagating in the negative $X$ direction also with speed $c$, where we recall that $c=\sqrt{g h}$, which here depends on the slow variable $\chi, c=c(\chi)$. We choose a wave propagating to the right, so that to leading order we get 


$$
\begin{aligned}
\zeta^{(1)} & =\frac{h}{c} U^{(1)}=A(\sigma, \theta), \\
\text { where } \quad T^{*} & =\int^{X} \frac{d X}{c}, \quad \theta=T^{*}-T, \quad \sigma=\epsilon^{2} T^{*} .
\end{aligned}
$$

Here we have anticipated that as the wave propagates to the right with speed $c$, it also evolves on the long spatial scale of $\epsilon^{-3}$, and so we have introduced the slow variable $\sigma$. Note that the either of the slow variables $\sigma$ or $\chi$ could be used here, but we have preferred the former as it has the dimensions of time.

At the next order we obtain the system of equations

$$
\begin{aligned}
\zeta_{T}^{(2)}+h U_{X}^{(2)} & =F^{(2)} \\
U_{T}^{(2)}+g \zeta_{X}^{(2)} & =G^{(2)} \\
\text { where } F^{(2)} & =-\zeta_{\sigma}^{(1)}-\frac{1}{c}\left(U^{(1)} \zeta^{(1)}\right)_{\theta}-\frac{c_{\sigma}}{c} \zeta^{(1)} \\
G^{(2)} & =-\frac{c}{h} \zeta_{\sigma}^{(1)}-\frac{1}{c} U^{(1)} U_{\theta}^{(1)}-\frac{h}{3 c} \zeta_{\theta \theta \theta}^{(1)} .
\end{aligned}
$$

Note that here, to leading order, $\zeta_{X}^{(1)}=\zeta_{\theta}^{(1)} / c$. From $(23)$ the inhomogeneous terms are function of $\sigma, \theta$, and so, to leading order this system of equations reduces to

$$
\begin{aligned}
& -c \zeta_{\theta}^{(2)}+h U_{\theta}^{(2)}=F^{(2)} \\
& -c U_{\theta}^{(2)}+g \zeta_{\theta}^{(2)}=G^{(2)}
\end{aligned}
$$

The homogeneous version of the system $(29,30)$ has a non-trivial solution, namely the right-propagating wave $\zeta^{(1)}, U^{(1)}$ given by $(23)$. Hence the inhomogeneous system $(29,30)$ can have a solution only if the inhomogeneous terms on the left-hand side are orthogonal to the non-trivial solution of the homogeneous adjoint system. This is readily found to be $(c, h)$ and so the required compatibility condition is

$$
c F^{(2)}+h G^{(2)}=0 .
$$

Next we substitute the expressions (23) into (31) and after some simplification get

$$
A_{\sigma}+\frac{c_{\sigma}}{2 c} A+\frac{3}{2 h} A A_{\theta}+\frac{h^{2}}{6 c^{2}} A_{\theta \theta \theta}=0 .
$$

Using the transformations $(17,24)$ and replacing $\epsilon^{2} A$ with $A$ this becomes

$$
A_{t}+c A_{x}+\frac{c_{x}}{2} A+\frac{3 c}{2 h} A A_{x}+\frac{h c^{2}}{6} A_{x x x}=0
$$

This is just the vKdV equation (9) for the case of water waves. Finally, using the transformation (10) with $Q=c$ equation (33) becomes 


$$
\begin{gathered}
\qquad \eta_{\tau}+\alpha^{*} \eta \eta_{\xi}+\beta^{*} A_{\xi \xi \xi}=0 \\
\text { where here } \quad \eta=\sqrt{c} A \quad \alpha^{*}=\frac{3}{2 h \sqrt{c}}, \quad \beta^{*}=\frac{h^{2}}{6 c^{2}} .
\end{gathered}
$$

The same type of multiscale asymptotic expansion can be used to derive a vKdV equation in many other physical systems. The key is the existence of a waveguide supporting a linear wave mode, whose dispersion relation for unidirectional sinusoidal waves, propagating along the waveguide (in the $x$ direction) with frequency $\omega$ and wavenumber $k$, has a long-wave expansion of the form.

$$
\omega=c k-\delta k^{3}+O\left(k^{5}\right) .
$$

A typical fluid variable, say $u(x, t, z)$ can then be represented in the form

$$
u=\epsilon^{2} A(\sigma, \theta) \phi(z)+\epsilon u^{(2)}+\cdots .
$$

Here the scaled variables $\sigma, \theta$ are again defined by $(24)$, and $\phi(z, \sigma)$ is a known modal function in the $z$-direction, where $z$ is a coordinate across the waveguide. For instance, for water waves and when $u$ is the amplitude of the free surface elevation, $\phi(z)=z / h(c=\sqrt{g h}$ in this case). But in most physical systems, the dependence on $z$ is not so simple, and is determined by an associated eigenvalue problem, which also determines the linear long-wave speed $c$. For instance, this is the situation for internal waves [5]. Note that in a slowly varying inhomogeneous medium, the modal function also depends parametrically on $\sigma$ (for water waves, through $h(\chi)$ ). It is immediately clear that for linearized waves in a homogeneous medium, the amplitude $A$ will satisfy the linearization of the $\mathrm{KdV}$ equation (1). The next task is to find the magnification factor $Q$ in (9) when the medium is inhomogeneous. This is most easily accomplished by finding the equation for conservation of wave action flux $Q A^{2}$ in the linear long wave regime, Thus the main task of the multiscale aysmptotic expansion is the determination of the nonlinear coefficient $\mu$. This is accomplished by constructing the equation for the second-order term in (37). This inevitably, as for water waves, takes the form of a linear inhomogeneous system, whose homogeneous part is just the defining equation for the linear long-wave mode being considered. Hence, the inhomogeneous system requires a compatibility condition, which yields the required KdV equation (9).

\section{Slowly-varying waves}

\subsection{Cnoidal waves}

Although our main concern will be with the behaviour of solitary waves, it is instructive to first review the asymptotic theory for slowly varying periodic waves, namely here the cnoidal waves defined by (3). In this case the theory is analogous to the well-known WKB procedure for linear waves. One can 
either use a multi-scale asymptotic expansion, or make use of appropriate conservation laws (see [4] for instance). Here we shall use the former approach, and so we now suppose that the coefficients $\alpha, \beta$ in (11) are slowly varying, and write

$$
\alpha=\alpha(S), \quad \beta=\beta(S), \quad S=\nu \tau, \quad Y=\nu \xi, \quad \nu<<1 .
$$

Here the slow temporal variable $S$ is introduced to provide an explicit description of the separation of scales between the variation of the coefficients and the more rapidly oscillating waves, while $Y$ is an analogous slow spatial variable. Next we seek a multi-scale expansion for a modulated periodic wave, namely

$$
\begin{aligned}
\eta & =\eta_{0}(\psi, S, Y)+\epsilon \eta_{1}(\psi, S, Y)+\cdots, \\
\text { where } \quad \psi & =\frac{1}{\nu} \Psi(S, Y) . \\
\text { and } \quad k & =\Psi_{Y}, k V=-\Psi_{S} .
\end{aligned}
$$

It is assumed that $\eta$ is periodic in $\psi$ with a fixed period of $2 \pi$. Equation (41) defines the local wavenumber $k$, the local frequency $k V$, and the local phase speed $V$. Cross-differentiation yields the equation for conservation of waves

$$
k_{S}+(k V)_{Y}=0 .
$$

We should recall here, that although we have called $k$ a wavenumber and $k V$ a frequency, they are not the actual phsyical wavenumber and frequency, because of the transformation of variables defined in (10). Indeed the physical wavenumber is $k(1-W) / c$, the physical frequency is $k$, and the physical phase speed is $c /(1-W) \approx c(1+W)$, since $W<<1$ due to the scaling used to derive the $\mathrm{KdV}$ equation (11)

Substitution of ( 39) into (11) yields, at the leading orders

$$
\begin{gathered}
-V \eta_{0 \psi}+\alpha \eta_{0} \eta_{0 \psi}+\beta k^{2} \eta_{0 \psi \psi \psi}=0 \\
-V \eta_{1 \psi}+\alpha\left(\eta_{0} \eta_{1}\right)_{\psi}+\beta k^{2} \eta_{1 \psi \psi \psi}=\frac{1}{k} F_{1}, \\
\text { where } \quad F_{1}=-\eta_{0 S}-\alpha \eta_{0} \eta_{0 Y}-3 \beta k^{2} \eta_{0 \psi \psi Y}-3 \beta k k_{Y} \eta_{0 \psi \psi} .
\end{gathered}
$$

Each of these is essentially an ordinary differential equation with $\psi$ as the independent variable, and with $S, Y$ as parameters. The solution of (43) is the cnoidal wave (see (3))

$$
\begin{aligned}
\eta_{0} & =a\left\{b(m)+\mathrm{cn}^{2}(\gamma \psi ; m)\right\}+d, \\
\text { where } \quad b(m) & =\frac{1-m}{m}-\frac{E(m)}{m K(m)}, \quad \alpha a=12 m \beta \gamma^{2} k^{2}, \\
\text { and } \quad V & =\alpha d+\frac{\alpha a}{3}\left\{\frac{2-m}{m}-\frac{3 E(m)}{m K(m)}\right\} .
\end{aligned}
$$


As before $\gamma=K(m) / \pi$, since $\eta_{0}$ is $2 \pi$-periodic in the phase $\psi$. In this solution the parameters $a, m, \gamma, V, d$ each depend on the slow variables $S, Y$ and now the task is to determine how they vary as functions of these slow variables. Note that there are three independent parameters, and hence three equations are needed. However, one of these is the conservation of waves equation (42). There are two main methods used to find the remaining two equations. One is the so-called Whitham averaging method, where one seeks two appropriate conservation laws for the $\mathrm{vKdV}$ equation (11), inserts the cnoidal wave (3) into these laws, and then averages over the phase $\psi$ (see [3], [4]). Here we shall first describe the second approach which is to continue the asymptotic expansion to the next order, and then invoke the condition that $A_{1}$ is a periodic function of $\psi$. It is implicit in the Whitham averaging procedure that the higher-order terms in the expansion have this property. Although it can be shown that the presence of a suitable underlying Lagrangian usually ensures that this is so (see [4]), we shall nevertheless verify it directly here for the first-order term. This is given by (44) in which the right-hand side is now a known periodic function of $\psi$, given by (45). A necessary and sufficient condition for $\eta_{1}$ to be periodic in $\psi$ is that the right-hand side of (44) should be orthogonal to the periodic solutions of the adjoint to the homogeneous operator on the left-hand side. This adjoint is

$$
-V \eta_{1 \theta}+\alpha \eta_{0} \eta_{1 \psi}+\beta k^{2} \eta_{1 \psi \psi \psi}=0 .
$$

It is readily seen that two solutions of (49) are $1, \eta_{0}$, both of which are periodic. A third solution can be found by the variation-of-parameters method, but it is not periodic. Hence there are two orthogonality conditons, given by

$$
\begin{gathered}
M_{0 S}+\left(\alpha P_{0}\right)_{Y}=0, \\
P_{0 S}+\left(\alpha Q_{0}\right)_{Y}=0, \\
M_{0}=\frac{1}{2 \pi} \int_{0}^{2 \pi} \eta_{0} d \psi=d, \quad P_{0}=\frac{1}{4 \pi} \int_{0}^{2 \pi} \eta_{0}^{2} d \psi, \\
Q_{0}=\frac{1}{6 \pi} \int_{0}^{2 \pi} \eta_{0}^{3} d \psi-\frac{3 \beta k^{2}}{4 \alpha \pi} \int_{0}^{2 \pi} \eta_{0 \psi}^{2} d \psi .
\end{gathered}
$$

As we discuss further below the first of these is the equation for conservation of "mass", and the second is an equation for conservation of "momentum". The final step is the substitution of $(46)$ into $(52,53)$, which gives

$$
\begin{aligned}
P_{0}= & \frac{d^{2}}{2}+\frac{a^{2}}{2}\left\{C_{4}-b^{2}\right\}, \\
Q_{0}= & \frac{d^{3}}{3}+d a^{2}\left\{C_{4}-b^{2}\right\}+\frac{a^{3}}{3}\left\{C_{6}-3 b C_{4}-2 b^{3}\right\} \\
& +\frac{3 \beta k^{2} \gamma^{2} a^{2}}{2 \alpha}\left\{m C_{6}-(2 m-1) C_{4}+(1-m) b\right\}
\end{aligned}
$$




$$
\begin{aligned}
C_{4} & =\frac{1}{K} \int_{0}^{K} \operatorname{cn}^{4}(u ; m) d u, \\
C_{6} & =\frac{1}{K} \int_{0}^{K} \operatorname{cn}^{6}(u ; m) d u, .
\end{aligned}
$$

Here we recall that $K(m)$ is the first elliptic integral, while $b(m)$ is defined in (47), and so $C_{4}(m), C_{6}(m)$ are functions of the modulus $m$ alone, given by

$$
\begin{aligned}
3 m^{2} C_{4}(m) & =\left(3 m^{2}-5 m+2\right)+(4 m-2) \frac{E(m)}{K(m)}, \\
15 m^{3} C_{6}(m) & =\left(15 m^{3}-34 m^{2}+27 m-8\right)+\left(23 m^{2}-23 m+8\right) \frac{E(m)}{K(m)}
\end{aligned}
$$

The alternative approach to the derivation of $(50,51)$ is to make direct use of the conservations laws for "mass" and "momentum",

$$
\begin{aligned}
\eta_{\tau}+\left\{\frac{\alpha}{2} \eta^{2}+\beta \eta_{\xi \xi}\right\}_{\xi} & =0, \\
\left(\frac{\eta^{2}}{2}\right)_{\tau}+\left\{\frac{\alpha}{3} \eta^{3}+\left\{\beta\left(\eta \eta_{\xi}\right)_{\xi}-\frac{3 \beta}{2} \eta_{\xi}^{2}\right\}_{\xi}\right. & =0 .
\end{aligned}
$$

Each of these is readily established from (11) and indeed (60) is just the vKdV equation (11) itself. Note that although we shall call these the laws for conservation of mass and momentum, the integrands do not necessarily correspond to the corresponding physical entities. Indeed, to leading order, (61) is usually the law for conservation of wave action flux. The conservation law (60) usually differs slightly from the actual law for conservation of mass. The issue has been explored by Miles [22] for water waves, where it can be shown that the difference is smaller than the error incurred in the derivation of the vKdV equation (33), and is due to reflected waves. The Whitham averaging procedure now consists of the substitution of $(46)$ into $(60,61)$, and then averaging the results over the phase (see [3], [4]). The result is readily seen to be the derived equations $(50,51)$.

The equation set $(42,50,51)$ are the three desired equations for the three chosen parameters, $(k, d, m)$ say, and form a nonlinear hyperbolic system, provided that the underlying periodic wave is stable. They are quite complicated, and in general it is difficult to find explicit solutions. The issue is present even when the coefficients $\alpha, \beta$ are constant. But in that case, due to the integrability of the KdV equation, it can be shown that a subtle change of variables leads to a set of three nonlinear hyperbolic equations in Riemann form, see [3], [4] for instance. The resulting equations are also integrable, through a generalized hodograph tarnsformation. However, in the general case when the coefficients $\alpha=\alpha(S), \beta=\beta(S)$, no such reduction is available. Instead the system remains coupled, although it can be cast into a more transparent form using the same Riemann variables available in the integrable case, see [20].

However, one situation of interest can be solved explicitly. Let us suppose that the solution set for $(k, d, m)$ depends only on $S$. Then equations (42, 
50) readily show that $k, M_{0}=d$ are respectively constants. The remaining variable $m$ is then found from the remaining equation (51), whose solution is $P_{0}=$ constant. Then, using $(54,56)$ and the relation $(47)$, we find that the amplitude $a$ and $m$ are related by the expression

$$
\left.\frac{a^{2}}{m^{2}}\left\{3 m^{2}-5 m+2\right)+(4 m-2) \frac{E(m)}{K(m)}-3 m^{2} b(m)^{2}\right\}=\text { constant } .
$$

Finally, using the relation (47) we can determine the modulus $m$ in terms of $\alpha(S), \beta(S)$,

$$
\begin{aligned}
& F(m)=\text { constant } \frac{\alpha^{2}}{\beta^{2}}, \quad \text { where } \\
& F(m)=K(m)^{2}\left\{(4-2 m) E(m) K(m)-3 E(m)^{2}-(1-m) K(m)^{2}\right\} .
\end{aligned}
$$

This expression for $F(m)$ was obtained by Ostrovsky and Pelinovsky [23], [24] and Miles [22] for the special case of water waves, where $\alpha / \beta=9 g^{3 / 4} / h^{9 / 4}$ (see $(35)) . F(m)$ is plotted in Figure 1, which shows that it is a monotonically increasing function of $m$. It follows that as $\alpha / \beta$ increases so does $m$. Two limiting situations are of interest. First, if the nonlinear coefficient $\alpha$ decreases towards zero, then so does the modulus $m$ where it can be shown that $F(m) \sim m^{2}$ as $m \rightarrow 0$; it follows that the modulus $m \sim \alpha$, but remarkably the amplitude $a$ is finite in this limit. On the other hand, if the dispersive coefficient $\beta \rightarrow 0$, then $m \rightarrow 1$ and the waves become more like solitary waves. For water waves, we see that this situation arises as the waves propagate into shallow water, that is $h \rightarrow 0$. In this limit, the expressions $(63,64)$ show that $a \sim K(m) \sim h^{-3 / 4}$. Recalling the transformation (35), this leads to the well-known result that a solitary wave propagating in shallow water behaves as $h^{-1}$, see [11], [23], [19].

\subsection{Solitary waves}

The results obtained above for a slowly-varying periodic wave cannot immediately be extrapolated to a slowly-varying solitary wave, as the limits $m \rightarrow 1$ and $\nu \rightarrow 0$ do not commute. In physical terms, the basis for the validity of the slowly-varying periodic wave is that the local wavelength (i.e. $2 \pi / k$ ) should be much less than the slowly-varying scale (i.e. $1 / \nu)$. The limit $m \rightarrow 1$ in $(46,47$, 48) requires that $\gamma \rightarrow \infty, k \rightarrow 0$ with $\gamma k=\Gamma$ held constant, and so the wavelength technically becomes much larger than the slow scale. A new concept of slowly-varying is needed, which in physical terms is that the half-width (i.e. width of the solitary wave at the level of one half of the maximum amplitude) should be much less than $1 / \nu$. We proceed as above and again invoke a multiscale asymptotic expansion of the same form (38.39) and we again obtain the equations $(43,44,45)$ (see, for instance, [12] [14]). Note that $k$ has a different meaning here, but that nevertheless equation (42) for conservation of waves 


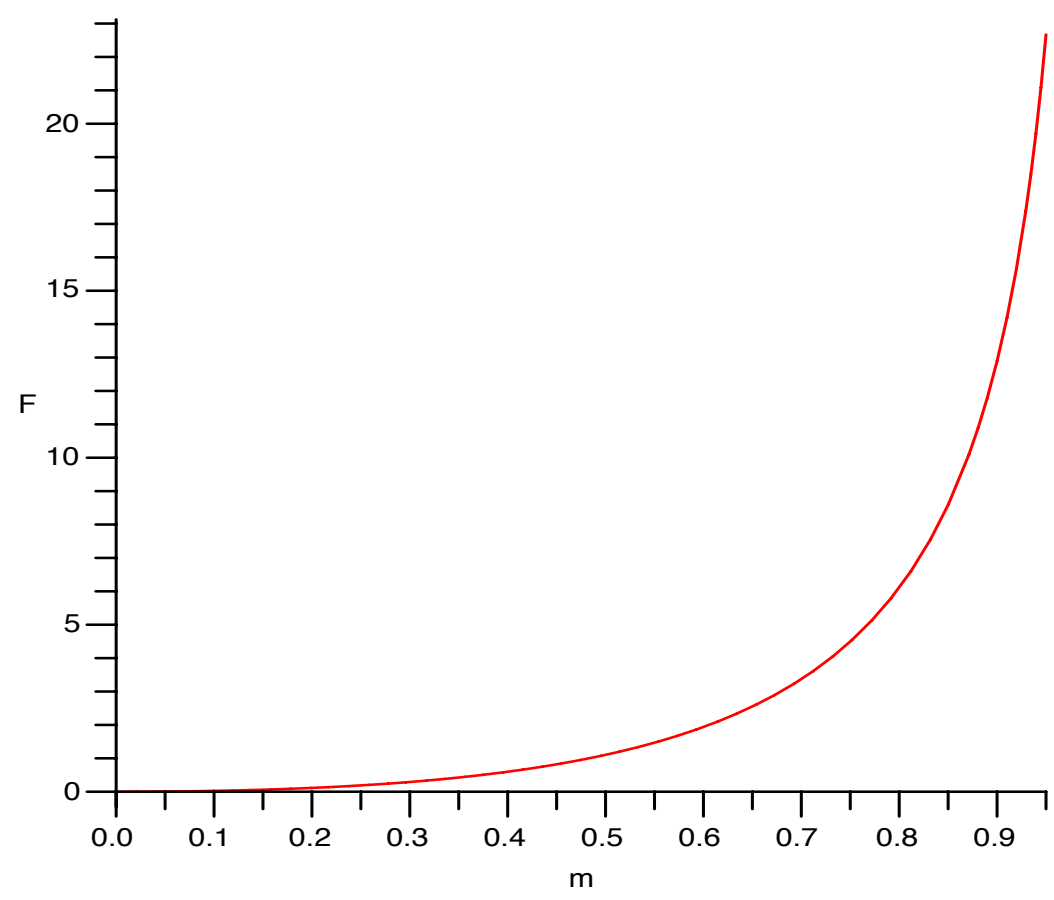

Fig. 1. A plot of $F(m)(64)$ versus $m$.

still holds. but now we do not require that $\eta$ is periodic in $\psi$ with a fixed period. Instead we require that $\eta$ is bounded in the limits $\psi \rightarrow \pm \infty$ (more strictly, we require that $\eta$ grows at infinity as $\nu^{m+1} \psi^{m}$ at each order $\nu^{m+1}$ for $m=0,1,2 \cdots)$. We can suppose without loss of generality that $\delta>0$, since the alternative case is recovered by replacing $\eta, \xi$ with $-\eta,-\xi$ respectively. Then, small-amplitude waves will propagate in the negative $\xi$-direction, and we can suppose that $\eta \rightarrow 0$ as $\psi \rightarrow \infty$. However, it will transpire that we cannot impose this boundary condition as $\psi \rightarrow-\infty$. Note that it is possible to allow $\eta \rightarrow D^{+}(S, Y)$ as $\psi \rightarrow \infty$ where $D^{+}(S, Y)$ is known a priori, and satisfies the vKdV equation (11), that is

$$
D_{S}^{+}+\alpha D^{+} D_{Y}^{+}+\nu^{2} \beta D_{Y Y Y}^{+}=0 .
$$

However, we shall not consider this extension here, although note that the basic procedure outlined below can be used also in this more general case.

Now the solution for $\eta_{0}$ is taken to be the solitary wave $(\operatorname{see}(7))$

$$
\begin{aligned}
A & =\operatorname{asech}^{2}(\Gamma \psi), \\
\text { where } \quad V & =\frac{\alpha a}{3}=4 \beta k^{2} \Gamma^{2} .
\end{aligned}
$$


Here there is just a single free parameter, one of the set $(a(S, Y), V(S, Y)$, $k \Gamma(S, Y)$ ), which depends on the slow variables. Its behaviour is determined by examining the next order equation (44), from which we seek a solution $\eta_{1}$ which is bounded as $\psi \rightarrow \pm \infty$ : as above we can require that $\eta_{1} \rightarrow 0$ as $\psi \rightarrow \infty$. As before, the adjoint equation to (44) is (49) for which two solutions are $1, \eta_{0}$; while both are bounded, only the second solution satisfies the condtion that $\eta_{1} \rightarrow 0$ as $\psi \rightarrow \infty$. A third solution can be constructed using the variation-of-parameters method, but it is unbounded as $\psi \rightarrow \pm \infty$. Hence only one othogonality condition can now be imposed, namely that the right-hand side of (44) is orthogonal to $\eta_{0}$, which leads to (51) where now

$$
\begin{aligned}
P_{0} & =\frac{1}{2} \int_{-\infty}^{\infty} \eta_{0}^{2} d \psi, \\
Q_{0} & =\frac{1}{3} \int_{-\infty}^{\infty} \eta_{0}^{3} d \psi-\frac{3 \beta k^{2}}{2 \alpha} \int_{-\infty}^{\infty} \eta_{0 \psi}^{2} d \psi .
\end{aligned}
$$

Substituting $(66,67)$ into $(68,69)$ yields

$$
P_{0}=\frac{2 a^{2}}{3 \Gamma}, \quad Q_{0}=\frac{2 a^{3}}{9 \Gamma} .
$$

Finally, substitution of these expressions into (51) yields

$$
P_{0 S}+\left(V P_{0}\right)_{Y}=o
$$

As the solitary wave (66) has just one free parameter (e.g. the amplitude $a$ ), this equation, together with (42) suffices to determine its variation.

We now recall that the $\mathrm{vKdV}$ equation (11) possesses two conservation laws, namely $(60,61)$ for mass and momentum respectively. But here we see that the equation (61), which reduces here to (71) for momentum is sufficient to determine the slowly-varying solitary wave. Hence we now see that the slowly-varying solitary wave cannot simultaneously conserve its mass. This is apparent when one examines the solution of (44) for $\eta_{1}$, from which it is readily shown that although $\eta_{1} \rightarrow 0$ as $\psi \rightarrow \infty, \eta_{1} \rightarrow D_{1}$ as $\psi \rightarrow-\infty$ where

$$
\begin{gathered}
-k V D_{1}=M_{0 S}^{s}+\left(\alpha P_{0}\right)_{Y}, \\
M_{0}^{s}=\int_{-\infty}^{\infty} \eta_{0} d \psi=\frac{2 a}{\Gamma} .
\end{gathered}
$$

Here $M_{0}^{s}$ can be interpreted as the mass of the solitary wave. It is readily verified that the right-hand side of (72) cannot vanish in general, and so the slowly-varying solitary wave generates a trailing shelf, whose amplitude at the rear of the solitary wave is $D_{1}$. This non-uniformity has been recognized for some time, see, for instance, [14] and the references therein. The trailing shelf $\eta_{s h}$ has small amplitude $O(\nu)$ but a long length-scale $O(1 / \nu)$, and so carries $O(1)$ mass, but $O(\nu)$ momentum. It resides behind the solitary wave, and to 
leading order has a value independent of $S$, so that $\eta_{s h}=\nu A_{1}(Y)$ for $\Psi<0$; it is determined by its value at the location $\Psi=0$ of the solitary wave, namely $A_{1}(\Psi=0)=D_{1}(72)$. It may readily be verified that the slowly-varying solitary wave and the trailing shelf together satisfy conservation of mass. The asymptotic expansion may be continued to second order [14]. At higher orders in $\nu$ the shelf itself will evolve and may generate secondary solitary waves [10]

The two equations $(42,51)$ may be uncoupled by defining

$$
\begin{aligned}
& \tilde{P}_{0}=\frac{P_{0}}{k}=\frac{2 a^{3}}{3 \Gamma k}=\sqrt{\frac{\beta a^{2}}{27 \alpha}}, \\
& \text { so that } \tilde{P}_{0 S}+V \tilde{P}_{0 Y}=0 .
\end{aligned}
$$

Here we have used (67) to show that $\tilde{P}_{0}$, the momentum per unit distance, is a function of the amplitude $a$ alone. Since $V=\alpha a / 3(67)$ we see that equation (74) is a single equation for the amplitude. It show that the amplitude deforms to conserve $\tilde{P}_{0}$, which propagates with the solitary wave speed $V$.

An important special case arises when there is no $Y$-dependence. In that case (42) shows that $k, \tilde{P}_{0}$ are both constants, and so (75) reduces to

$$
a^{3}=\operatorname{constant} \frac{\alpha}{\beta}, \quad \text { or } \quad(\Gamma k)^{3}=\operatorname{constant} \frac{\alpha^{2}}{\beta^{2}} .
$$

In this same special case, $(72)$ reduces to

$$
V k \Gamma D_{1}=2 a_{s} .
$$

The expression (76) shows that the amplitude increases (decreases) in absolute value as $\alpha / \beta$ increases (decreases). Note that the polarity of the wave is determined by the sign of $\alpha / \beta$. Then, assuming without loss of generality that $\beta>0$ so that $V>0$, we see from (77) that a slowly-varying solitary wave of increasing (decreasing) amplitude, will generate a trailing shelf of the same (opposite) polarity. A particular case of interest is when the nonlinear coefficient $\alpha$ passes through zero, while $\beta$ stays finite. Suppose this occurs at $S=0$, where, without loss of generality, we may suppose that $\alpha$ passes from positive to negative values as $S$ increases. Initially the solitary wave is located in $S<0$ and has positive polarity. Then, near the transition point, the amplitude of the wave decreases to zero as $a \sim \alpha^{1 / 3}$, while $\Gamma \sim \alpha^{2 / 3}$; the momentum of the solitary wave is of course conserved (at least to leading order), the mass of the solitary wave increases as $1 / \alpha^{1 / 3}$, its speed decreases as $\alpha^{4 / 3}$, and the amplitude $D_{1}$ of the trailing shelf just behind the solitary wave grows as $-1 / \alpha^{8 / 3}$; the total mass of the trailing shelf grows as $-1 / \alpha^{1 / 3}$, in balance with that of the solitary wave, while the total mass remains a positive constant. Thus the solitary wave itself is destroyed as the wave attempts to pass through the critical point $\alpha=0$. The structure of the solution beyond this critical point has been examined numerically by Grimshaw et al [15], who showed that, in essence, the shelf passes through the critical point as a 
negative disturbance, which then being in an environment with $\alpha<0$, can generate a train of solitary waves of negative polarity, riding on a positive pedestal. Of course, these conclusions may need to be modified when a cubic nonlinear term in is taken into account near the critical point (see Grimshaw et al [16]).

For the special case of water waves, $\alpha / \beta=9 g^{3 / 4} / h^{9 / 4}$ (see (35)) and so the amplitude behaves as $a \sim h^{-3 / 4}$, while the speed $V$ behaves as $h^{-2}$, and the wavenumber $\Gamma$ behaves as $h^{-3 / 2}$. Recalling the transformation (10 where $Q=c=(g h)^{1 / 2}$ for water waves, we recover the well-known result that the surface elevation amplitude of a surface solitary wave behaves as $h^{-1}$ (see [11], [19], [23]). At the same time we see from (77) that the trailing shelf has negative polarity and behaves as $-h^{2}$ at the rear of the solitary wave.

\section{Soliton fission}

In the previous section we have considered the case when the variable coefficients in (11) vary slowly relative to a solitary wave. Here we consider the opposite case when the coefficients vary rapidly relative to a solitary wave. This scenario was considered by Johnson [19] for water waves and by Djordjevic and Redekopp [9] for internal waves. Let us suppose therefore that

$$
\begin{gathered}
\alpha=\alpha(Z), \beta=\beta(Z), \tau=\Delta Z, \Delta<<1, \\
\text { where } \alpha(Z) \rightarrow \alpha_{a, b}, \beta(Z) \rightarrow \beta_{a, b}, \text { as } Z \rightarrow \pm \infty,
\end{gathered}
$$

Thus, effectively the vKdV equation (11) has constant coefficients in $\tau<0$ and in $\tau>0$, while there is a small transition region in which $\tau$ is $O(\Delta)$ where the coefficients change their values from $\alpha_{b}, \beta_{b}$ in $\tau<0$ to $\alpha_{a}, \beta_{a}$ in $\tau>0$. In this transition zone, we may write $\eta=\eta(Z, \xi)$, and it is then readily shown that $\eta_{Z}$ is $O(\Delta)$. Hence, to leading order, it follows that $\eta$ is unchanged in the transition zone.

We now suppose that there is a single solitary wave in $\tau<0($ see $(7,8))$,

$$
\begin{aligned}
A & =a \operatorname{sech}^{2}(\Gamma(\xi-V \tau)), \\
\text { where } \quad V & =\frac{\alpha_{b} a}{3}=4 \beta_{b} \Gamma^{2} .
\end{aligned}
$$

This wave will pass through the transition zone unchanged, but, on arrival into the region $\tau>0$ it is no longer a permissible solution of (11), which now has constant coefficients $\alpha_{a}, \beta_{b}$. Instead, with $\tau=0+$, the expression (80) forms an effective initial condition for the new constant-coefficient KdV equation. Using the inverse scattering transform, the solution in $\tau>0$ can now be constructed; indeed in this case there is an explicit solution (e.g. [2]). The outcome is that the initial solitary wave fissions into $N$ solitons, and some radiation. The number $N$ of solitons produced is determined by the ratio of coefficients $R=\alpha_{a} \beta_{b} / \alpha_{b} \beta_{a}$. If $R>0$ (i.e. there is no change 
in polarity for the solitary waves), then $N=1+[(\sqrt{8 R+1}-1) / 2]([\cdots]$ denotes the integral part); as $R$ increases from 0 , a new soliton (initially of zero amplitude) is produced as $R$ successively passes through the values $m(m+1) / 2)$ for $m=1,2, \cdots$. But if $R<0$ (i.e. there is a change in polarity) no solitons are produced and the solitary wave decays into radiation.

For instance, for water waves $\alpha=3 /\left(2 h c^{1 / 2}\right), \beta=h^{2} /\left(6 c^{2}\right)$ (35) where $h$ is the water depth. It can then be shown that a solitary water wave propagating from a depth $h_{b}$ to a depth $h_{a}$ will fission into $N$ solitons where $N$ is given as above with $R=\left(h_{b} / h_{a}\right)^{9 / 4}$. Here $R>0$, and it follows that if $h_{b}>h_{a}$, so that the solitary wave moves into shallower water, $N \geq 2$ and at least one more soliton is produced; the initial soliton is said to have fissioned. But if $h_{b}<h_{a}$ so that the solitary wave moves into deeper water, then $N=1$ and no further solitons are produced [19].

\section{References}

1. Ablowitz, M. and H. Segur, H. (1981) Solitons and the Inverse Scattering Transform. SIAM Studies in Applied Mathematics 4, Philadeplhia.

2. Drazin, P.G. and Johnson, R.S. (1989). Solitons: an Introduction. Cambridge University Press, Cambridge.

3. Kamchatnov, A.M. (2000) Nonlinear Periodic Waves and Their Modulations: An Introductory Course. World Scientific, Singapore.

4. Whitham, G.B. (1974). Linear and Nonlinear Waves, Wiley, New York.

5. Grimshaw, R. (2001) Internal solitary waves, in "Environmental Stratified Flows" . Kluwer, Boston, Chapter 1, 1-28.

6. P. Holloway, E. Pelinovsky and T. Talipova 2001. Internal tide transformation and oceanic internal solitary waves. In "Environmental Stratified Flows" (Kluwer, Boston, Chapter 2: 29-60, 2001).

7. J. Rottman and R. Grimshaw. Atmospheric internal solitary waves. In "Environmental Stratified Flows" (Kluwer, Boston, Chapter 3: 61-88, 2001).

8. Boussinesq. M.J. (1871) Theórie de l'intumescence liquide appellée onde solitaire ou de translation, se propageant dans un canal rectangulaire. Comptes Rendus Acad. Sci (Paris), 72, 755-759.

9. Djordjevic, V. D. and Redekopp, L.G. (1978). The fission and disintegration of internal solitary waves moving over two-dimensional topography. J. of Phys. Ocean., 8, 1016-1024.

10. El, G.A. and Grimshaw, R. (2002) Generation of undular bores in the shelves of slowly-varying solitary waves. Chaos, 12, 1015-1026.

11. Grimshaw, R. (1970). The solitary wave in water of variable depth. J. Fluid Mech., 42, 639-656.

12. Grimshaw, R. (1979) Slowly varying solitary waves. I Korteweg-de Vries equation. Proc. Roy. Soc., 368A, 359-375.

13. Grimshaw, R. (1981) Evolution equations for long nonlinear internal waves in stratified shear flows. Stud. Appl. Math., 65, 159-188.

14. Grimshaw, R. and Mitsudera, H. (1993) Slowly-varying solitary wave solutions of the perturbed Korteweg-de Vries equation revisited. Stud. Appl. Math., 90, $75-86$. 
15. Grimshaw, R., Pelinovsky, E. and Talipova, T. (1998) Solitary wave transformation due to a change in polarity. Stud. Appl. Math., 101, 357-388.

16. Grimshaw, R., Pelinovsky, E. and Talipova, T. (1999). Solitary wave transformation in a medium with sign-variable quadratic nonlinearity and cubic nonlinearity. Physica D, 132, 40-62.

17. R. Grimshaw, E. Pelinovsky, T. Talipova, T. and A. Kurkin. Simulation of the transformation of internal solitary waves on oceanic shelves. J. Phys. Ocean., 34, 2774-2779 (2004).

18. Gurevich, A. V. and Pitaevskii, L. P. (1974) Nonstationary structure of a collisionless shock wave. Sov. Phys. JETP 38, 291-297.

19. Johnson, R.S. (1973) On the development of a solitary wave moving over an uneven bottom. Proc. Camb. Phil. Soc., 73, 183-203.

20. Kamchatnov, A.M. (2004). On Whitham theory for perturbed integrable equations, Physica D, 188, 247-261 (2004).

21. Korteweg, D.J. and de Vries, H. (1895) On the change of form of long waves advancing in a rectangular canal, and on a new type of long stationary waves. Philosophical Magazine, 39, 422-443.

22. Miles, J.W. (1979) On the Korteweg-de Vries equation for a gradually varying channel. J. Fluid Mech., 91, 181-190.

23. Ostrovsky, L.A. and Pelinovsky, E.N. (1970) Wave transformation on the surface of a fluid of variable depth. Akad. Nauk SSSR, Izv. Atmos. Ocean Phys., 6, 552-555.

24. Ostrovsky, L.A. and Pelinovsky, E.N. (1975). Refraction of nonlinear sea waves in a coastal zone. . Akad. Nauk SSSR, Izv. Atmos. Ocean Phys., 11, 37-41.

25. Lord Rayleigh (1876) On waves. Phil. Mag. 1, 257-279.

26. Russell, J.S. (1844) Report on Waves. 14th meeting of the British Association for the Advancement of Science, 311-390.

27. Zabusky, N.J. and Kruskal, M.D. (1965). Interactions of solitons in a collisionless plasma and the recurrence of initial states. Physical Review Letters, 15 240-243. 